Article

\title{
Silymarin-Loaded Nanoparticles Based on Stearic Acid-Modified Bletilla striata Polysaccharide for Hepatic Targeting
}

\author{
Yanni Ma ${ }^{1}$, Shaolong $\mathrm{He}^{2}{ }^{2}$, Xueqin Ma ${ }^{2}$, Tongtong Hong ${ }^{3}$, Zhifang Li $^{2}$, Kinam Park ${ }^{4}$ and \\ Wenping Wang ${ }^{2, *}$
}

1 Institute of Clinical Pharmacology, General Hospital of Ningxia Medical University, Yinchuan, Ningxia 750004, China; yannima2015@sina.com

2 Department of Pharmaceutics, School of Pharmacy, Ningxia Medical University, 1160 Shengli Street, Yinchuan, Ningxia 750004, China; 15296907978@163.com (S.H.); xueqinma2015@126.com (X.M.); lizhifang2015@sina.com (Z.L.)

3 Department of Pharmacy, General Hospital of Yankuang Group, Zou Cheng, Shandong 273500, China; tongtonghong2015@sina.com

4 Departments of Biomedical Engineering and Pharmaceutics, Purdue University, West Lafayette, IN 47907, USA; kpark@purdue.edu

* Correspondence: wpwang2015@126.com; Tel.: +86-951-688-0581; Fax: +86-951-688-0693

Academic Editor: Derek J. McPhee

Received: 18 January 2016 ; Accepted: 22 February 2016 ; Published: 29 February 2016

\begin{abstract}
Silymarin has been widely used as a hepatoprotective drug in the treatment of various liver diseases, yet its effectiveness is affected by its poor water solubility and low bioavailability after oral administration, and there is a need for the development of intravenous products, especially for liver-targeting purposes. In this study, silymarin was encapsulated in self-assembled nanoparticles of Bletilla striata polysaccharide (BSP) conjugates modified with stearic acid and the physicochemical properties of the obtained nanoparticles were characterized. The silymarin-loaded micelles appeared as spherical particles with a mean diameter of $200 \mathrm{~nm}$ under TEM. The encapsulation of drug molecules was confirmed by DSC thermograms and XRD diffractograms, respectively. The nanoparticles exhibited a sustained-release profile for nearly 1 week with no obvious initial burst. Compared to drug solutions, the drug-loaded nanoparticles showed a lower viability and higher uptake intensity on HepG2 cell lines. After intravenous administration of nanoparticle formulation for $30 \mathrm{~min}$ to mice, the liver became the most significant organ enriched with the fluorescent probe. These results suggest that BSP derivative nanoparticles possess hepatic targeting capability and are promising nanocarriers for delivering silymarin to the liver.
\end{abstract}

Keywords: silymarin; Bletilla striata; polysaccharide; nanoparticles; hepatic targeting

\section{Introduction}

Silymarin (SM) is a flavonolignan mixture derived from the seeds of Silybum marianum L. Gaertn (milk thistle), with silybin as its main component (70\%-80\%) [1]. SM has been widely used as a hepatoprotective agent for a variety of acute and chronic liver diseases, due to its therapeutic effect based on its antioxidant [2], anti-inflammatory [3], immunomodulatory [4] and anti-viral activities [5]. Previous reports have shown that the efficacy of oral SM on liver function and chronic hepatitis $C$ virus (HCV) load was low, due to its low bioavailability, extensive first pass metabolism and short life of flavonoids [6]. However, intravenous (i.v.) SM was well tolerated and exhibited a substantial antiviral 
effect against in non-responders [7]. Thus, introducing SM as a safe and efficient i.v. formulation is a major challenge. A few attempts have been made to explore the parenteral administration of SM. Christodoulou et al. developed a water-soluble silibinin-hydroxypropyl- $\beta$-cyclodextrin lyophilized product, but drug level rapidly decreased in serum and drug distribution in heart, kidney and liver was similar after i.v. administration $(20 \mathrm{mg} / \mathrm{kg}$ ) to mice [8]. In the work of Yliperttula's group, $\beta$-sitosterol $\beta$-D-glucoside (Sito-G) was added to the liposome formulation to enhance the uptake of SM in HepG2 cells, yet the in vivo performance was not determined [9]. Therefore, further studies on novel drug delivery system are required for liver-targeting and prolonged therapeutic effect.

Amphiphilic polymers can associate into nanoscaled interpolymeric self-aggregates with a hydrophobic core and a hydrophilic shell in aqueous media. Among these polymers, hydrophobically-modified polysaccharides have attracted much attention due to their biodegradability and biocompatibility, and their potential application as drug vectors has been widely explored, especially for delivery of poorly soluble drugs [10-12]. Moreover, self-assembled nanoparticles with targeting ligands are promising carriers for anti-cancer drugs, which are aimed are providing enhanced therapeutic efficacy and reduced systematic toxicity [13]. However, the conjugation of polymers with targeting agents usually involves a complex procedure and induces a decreased yield of derivatives. Sometimes polysaccharides themselves can act as active agents due to their bioactivity, and hyaluronic acid is a typical example [14]. Most commonly used polysaccharides include pullulan, chitosan, cellulose, dextran, heparin and hyaluronan [15]. Other natural polysaccharides are seldom investigated to develop micelle systems.

Bletilla striata polysaccharide (BSP), as an extract from the tubers of Bletilla striata, is a neutral water-soluble glucomannan with a backbone of $(1 \rightarrow 4)$-linked $\beta$-D-mannose and glucose in a molar ratio of 3:1. In our previous study [16], fatty acids with different chain lengths were used to modify BSP, and the BSP hydrophobically modified by stearic acid (hm-BSP) showed a preferable self-assembly property, as well as, good biocompatibility.

The aim of the present work was to evaluate the potential application of the stearic acid modified-BSP micelles for liver delivery of SM. SM-loaded nanoparticles based on hm-BSP were prepared and their morphology, particle size, thermal and crystalline properties characterized, and their in vitro release studied as well. The HepG2 cell line was applied to evaluate the cytotoxicity and cell uptake of the obtained nanoparticles, and biodistribution in mice after intravenous administration of nanoparticle and solution formulations was also observed under an imaging system.

\section{Results and Discussion}

\subsection{Preparation and Characterization of Silymarin-Loaded Nanoparticles}

Figure 1 shows the size distribution and morphology of drug-loaded nanoparticles by DLS and TEM, respectively. SM-hm-BSP nanoparticles were almost spherical in shape under TEM. Their particle size was around $180 \mathrm{~nm}$ when observed under TEM and $200 \mathrm{~nm}$ as determined by DLS, which is mainly due to the different preparation processes of the samples. TEM depicts the size of the samples in a dried state, whereas, DLS depicts the size in the hydrated state.

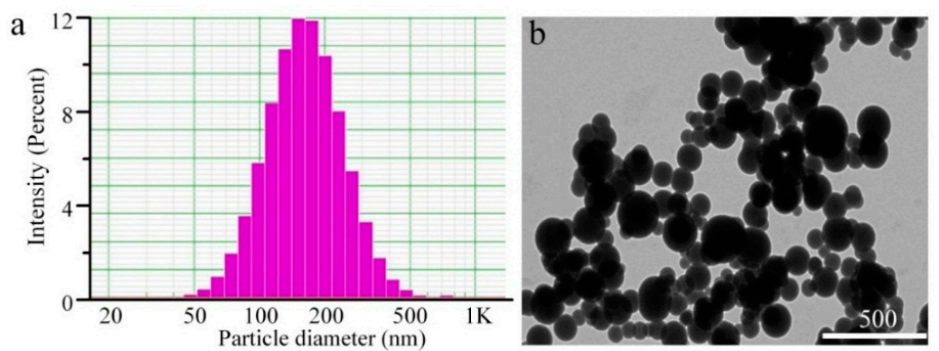

Figure 1. (a) Particle size distribution and (b) TEM image of SM-hm-BSP nanoparticles. 
According to our previous work, the average size of empty nanoparticles was $250 \mathrm{~nm}$ [16], which was smaller than that of the drug-loaded nanoparticles. The reason might be the encapsulation of a hydrophobic drug leads to a more compact core-shell structure.

Table 1 summarizes the characteristics of the SM-hm-BSP nanoparticles. The zeta potential of the nanoparticles was nearly neutral in water, and the EE of silymarin-loaded nanoparticles was approximately $80 \%$ at a theoretical DL of $10 \%$.

Table 1. Physicochemical properties of SM-hm-BSP nanoparticles.

\begin{tabular}{llllll}
\hline Sample & Diameter $(\mathbf{n m})$ & PDI & ${\text { Zeta }(\mathbf{m V})^{\text {a }}}$ & EE (\%) & DL (\%) \\
\hline SM-hm-BSP & $200.83 \pm 8.10$ & $0.25 \pm 0.04$ & $-0.36 \pm 0.93$ & $78.86 \pm 0.66$ & $7.31 \pm 0.05$ \\
\hline \multicolumn{5}{c}{ The zeta potential of micelles in distilled water at $1.50 \mathrm{mg} / \mathrm{mL}}$.
\end{tabular}

DSC thermograms of drug, polymer, physical mixture and drug-loaded nanoparticles are illustrated in Figure 2. The polymer showed a wide endothermic peak at $80^{\circ} \mathrm{C}$. SM powder showed a sharp endothermic peak at about $165^{\circ} \mathrm{C}$ corresponding to its melting point and then decomposed above $270{ }^{\circ} \mathrm{C}$, which was also observed by Jia et al. [17]. The peaks of drug and polymer were retained in the curve of their physical mixture. In contrast, no melting peak corresponding to the fusion of SM was observed in the curve of drug-loaded nanoparticles, indicating a loss of crystallinity after encapsulation in the hm-BSP nanocarriers.

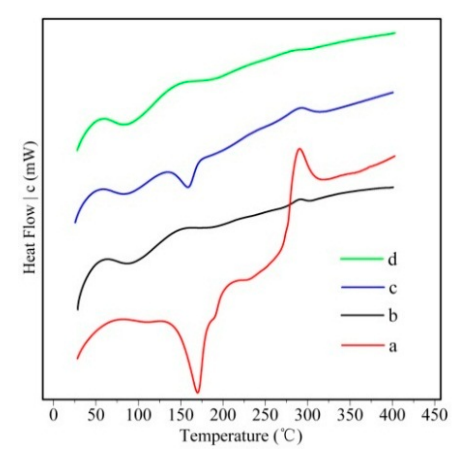

Figure 2. DSC thermograms of (a) SM; (b) hm-BSP; (c) physical mixture and (d) SM-hm-BSP nanoparticles.

To further confirm that SM was successfully loaded into the hm-BSP nanoparticles, XRD patterns of drug, polymer, the physical mixture and drug-loaded nanoparticles were recorded and are shown in Figure 3.

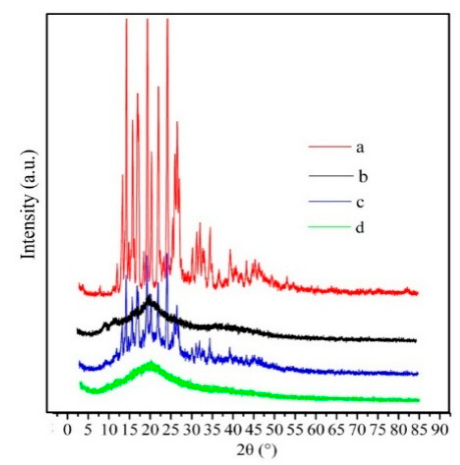

Figure 3. XRD patterns of (a) SM; (b) hm-BSP; (c) physical mixture and (d) SM-hm-BSP nanoparticles. 
It can be observed that the XRD spectra show broad peaks in the hm-BSP. The major peaks of crystalline SM (at $2 \theta$ range of $12^{\circ}$ to $35^{\circ}$ ) were detected in diffractograms of SM powder [18], and the physical mixture as well. However, the specific peaks of SM crystals disappeared in the drug-loaded nanoparticles, indicating that SM was molecularly dispersed within the nanocarriers. These results indicate that SM was successfully encapsulated into the nanoparticles as a molecular dispersion [19].

\subsection{In Vitro Release Study}

The in vitro drug release behavior of SM-hm-BSP nanoparticles was determined in $\mathrm{pH} 7.4$ PBS at $37 \pm 0.5^{\circ} \mathrm{C}$. As shown in Figure 4, the SM-hm-BSP nanoparticles showed a sustained release pattern with no obvious initial burst release. It follows a typical drug release from a matrix formulation, in which the drug release in the beginning is higher than that of later times. The steady release state lasted for almost one week and up to $95 \%$ of the drug was released by the end of the experiment. The slow drug release may have resulted from the electrostatic interaction between the drug and self-assembled micelles [20]. The week-long release of silymarin is interesting for a few reasons. First, it is not known whether the in vitro drug release is reproduced in vivo. Most i.v. administered drug delivery systems have not been examined for their in vivo drug release properties. Assuming the week-long release in vivo, however, it presents another related question. Since it is known that nanoparticles will not circulate in the blood for a week, the usefulness of the week-long drug release remains to be seen. It is possible, however, that the nanoparticles accumulated in the liver may slowly release the drug over a period of a week, and this may present a new way of drug delivery to the target for effectiveness lasting a week or more.

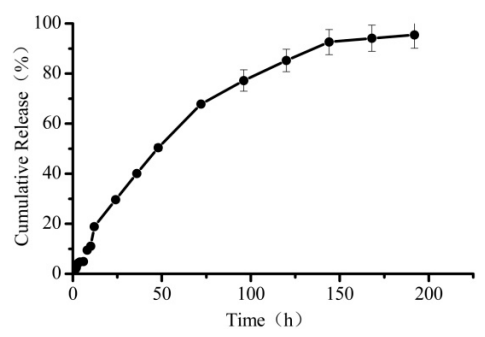

Figure 4. In vitro drug release profiles from SM-hm-BSP nanoparticles in pH 7.4 PBS.

\subsection{Cytotoxicity Test}

The cytotoxicity of SM and SM-hm-BSP on HepG2 cells at different intervals was evaluated through the CCK-8 assay. As shown in Figure 5, the inhibitory effect of free SM and the SM-hm-BSP nanoparticles on cell proliferation increased over a time period of $72 \mathrm{~h}$. and the cell suppression of free drug and drug-loaded nanoparticles against HepG2 cell lines exhibited a dose-dependent effect. Compared to SM solution $\left(\mathrm{IC}_{50}=7.50 \mu \mathrm{g} / \mathrm{mL}\right)$, SM-hm-BSP nanoparticles exhibited enhanced growth inhibition effects on HepG2 cells in almost all concentrations in $72 \mathrm{~h}$, and showed a significantly lower $\mathrm{IC}_{50}$ value $(0.66 \mu \mathrm{g} / \mathrm{mL})$. These findings indicate that the pharmacological activity of SM was enhanced after encapsulated into hm-BSP nanoparticles.
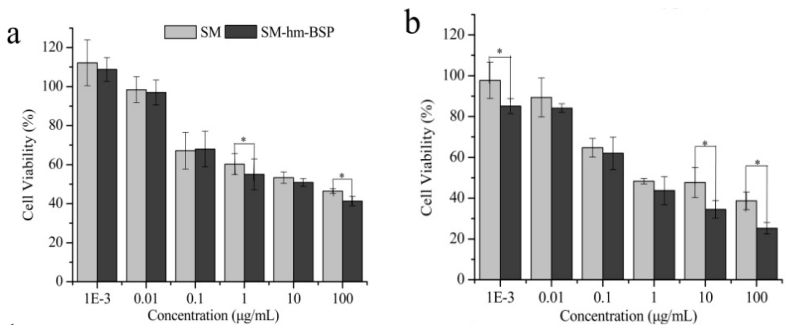

Figure 5. Cont. 


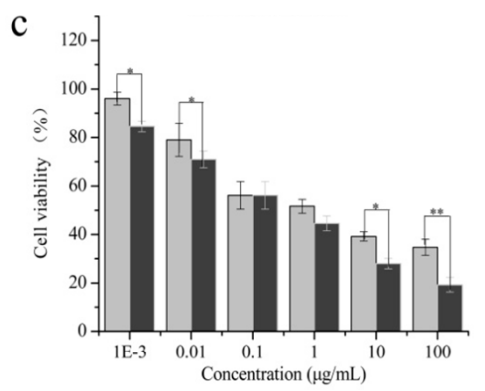

Figure 5. Cell viability of HepG2 cells treated with various concentration of SM and SM-hm-BSP nanoparticles for (a) $24 \mathrm{~h}$; (b) $48 \mathrm{~h}$ and (c) $72 \mathrm{~h} .(n=6) .{ }^{*} p<0.05$; ${ }^{* *} p<0.01$.

It is interesting that SM-hm-BSP nanoparticles had much better anti-cancer cell proliferation activity than free SM itself. Although drug release from the nanoparticles was slow. SM-hm-BSP nanoparticles showed a significant inhibition effect on HepG2 cell proliferation compared to the SM solution. Since hm-BSP itself was proven to be a preferable cytocompatibility material [16], we assumed that the main reason for the difference in cytotoxicity was that the micelles had a high affinity for hepatocytes. To confirm this hypothesis, the cellular internalization capacity of HepG2 cells for free C6 and C6-hm-BSP nanoparticles were further investigated by fluorescence microscopy and fluorometry, respectively.

\subsection{Cell Uptake and Flow Cytometry Study}

Figure 6 shows the fluorescence microscopy images of HepG2 cells following incubation with the free C6 and C6-hm-BSP nanoparticles for $1 \mathrm{~h}$. Cells incubated with the medium (control) showed almost no fluorescence (Figure 6a), and those with the free $\mathrm{C} 6$ solution had a weak fluorescence (Figure 6b). In contrast, a strong fluorescence was observed in the cells after incubation with the C6-hm-BSP nanoparticles (Figure 6c), indicating that C6 in C6-hm-BSP nanoparticles had an improved cellular uptake. The mean fluorescence intensity of the cells treated with the free C6 and C6-hm-BSP nanoparticles was quantitatively analyzed by a flow cytofluorometer. As shown in Figure 7, the concentration of C6 in HepG2 cells treated with C6-hm-BSP nanoparticles was 2.4-fold higher than that in cells incubated with free C6. Thus, hm-BSP nanoparticles achieved an excellent drug delivery effect, which is in agreement with the earlier results that fatty acid modified glucomannans have the potential to act as gene delivery vectors [21].
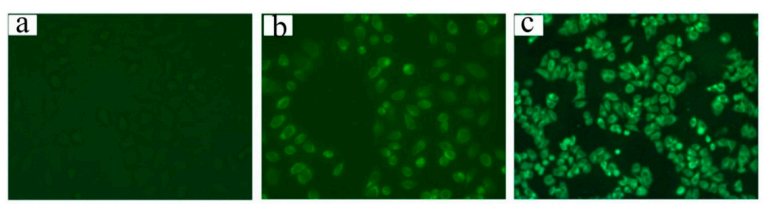

Figure 6. Fluorescence images $(\times 200)$ of HepG2 cells treated with $(\mathbf{a})$ medium; $(\mathbf{b})$ free C6 and (c) C6-loaded nanoparticles for $1 \mathrm{~h}$. The C6 content in micelle solutions was $0.089 \mu \mathrm{g} / \mathrm{mL}$.

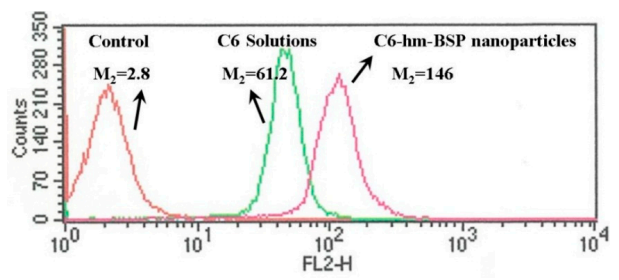

Figure 7. Fluorescence intensities of HepG2 cells treated with medium, free C6 and C6-loaded nanoparticles for $1 \mathrm{~h}$. The C6 content in micelle solutions was $0.089 \mu \mathrm{g} / \mathrm{mL}$. 


\subsection{Tissue Distribution Study}

As shown in Figure 8a, a nonspecific distribution of a fluorescent signal all over the body was observed after treatment with DIR solution for $30 \mathrm{~min}$. In contrast, a strong signal was shown only in the liver region of the live mice after injection with DIR-loaded nanoparticles, suggesting that the fluorescence probe was mostly accumulated in the liver. The major organs were then removed and analyzed directly under the imager (Figure 8b). A decreased fluorescence intensity for DIR-loaded nanoparticles was observed in the lung, heart and kidney, in comparison with that for DIR solution. However, the fluorescence signal of DIR nanoparticles (2261.4) in the liver was 17.2-fold higher than that of free DIR (131.4), indicating that hm-BSP nanocarriers could selectively deliver more DIR molecules into the liver.

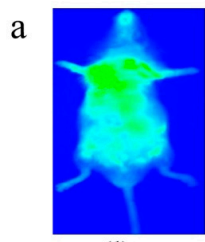

(1)

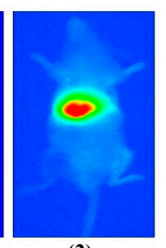

(2)
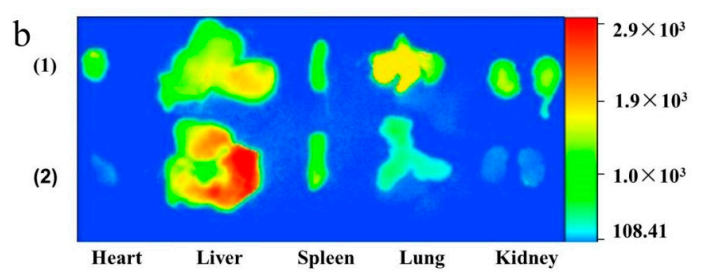

Figure 8. Fluorescence images of (a) mice body; (b) major organs of DIR after i.v. administration of DIR Solution (1) and DIR-labeled nanoparticles (2) into mice for $30 \mathrm{~min}$.

Taken together, the above results suggest that the hm-BSP nanoparticles will be promising liver-targeted delivery vectors, especially for hepatoprotective drugs with poor aqueous solubility. On the other hand, it also provides a reference for the development and application of functionalized polymers based on natural polysaccharides.

\section{Experimental Section}

\subsection{Materials}

Silymarin ( $\geqslant 98 \%$ ) and coumarin 6(C6) were purchased from Sigma-Aldrich (St. Louis, MO, USA). 1,1'-Dioctadecyl-3,3,3', , $3^{\prime}$-tetramethylindotricarbocyanine iodide (DIR) was bought from Amyjet Scientific Inc. (Wuhan, China). N,N'-Dicyclohexylvcarbodiimide (DCC) was acquired from Bioduly (Nanjing, China) and dimethylaminopyridine (DMAP) was from Kelong Chemical Regent Co., Ltd. (Chengdu, China). Phosphoric acid was obtained from Chemical Company (Tianjin, China). Polyethylene glycol (PEG400) was purchased from Shanghai Chemical Reagent Co., Ltd. (Shanghai, China). Dialysis tubing (molecular weight cut-off $3.4 \mathrm{kD}$ ) was supplied by Greenbird Biological Technology Co., Ltd. (Shanghai, China). Dulbecco's Modified Eagle's Medium (DMEM) and fetal calf serum (FCS) were obtained from Thermo Fisher Scientific, Inc. (Waltham, MA, USA). The tetrazolium-8-[2-(2-methoxy-4-nitrophenyl)-3-(4-nitrophenyl)-5-(2,4-disulfophenyl)-2H-tetrazolium] monosodium salt (CCK-8) was purchased from Dojindo Laboratories (Kumamoto, Japan). Methanol and acetonitrile were of HPLC grade. Phosphate-buffered saline (PBS; $0.01 \mathrm{M}, \mathrm{pH}$ 7.2-7.6) and other reagents were of analytical grade and used as received. Double distilled water was prepared in our laboratory.

\subsection{Synthesis of $h m-B S P$}

The hm-BSP derivative was synthesized according to the method reported by Sallustio et al. [22]. Briefly, BSP $(0.100 \mathrm{~g})$ was dissolved in DMSO $(20 \mathrm{~mL})$. Stearic acid $(0.284 \mathrm{~g})$ was activated by the addition of DCC $(0.206 \mathrm{~g})$ and DMAP $(0.146 \mathrm{~g})$ and dropped into BSP solution while stirring. The mixture was continuously stirred for $2 \mathrm{~h}$ at $80^{\circ} \mathrm{C}$, and then for $24 \mathrm{~h}$ at room temperature. The resulting solution was further dialyzed (molecular weight cut-off $3.4 \mathrm{kD}$ ) against water and freeze-dried to obtain the final product of hm-BSP. 


\subsection{Preparation of Silymarin-Loaded Nanoparticles}

SM-loaded nanoparticles were prepared by an ultrasonication dispersion method [23,24]. Briefly, $\mathrm{hm}-\mathrm{BSP}(25.0 \mathrm{mg})$ was dispersed in water at a concentration of $2.5 \mathrm{mg} / \mathrm{mL}$, and then slowly treated dropwise with $0.5 \mathrm{~mL}$ drug solution in ethanol $(5.0 \mathrm{mg} / \mathrm{mL})$ followed by stirring at room temperature for $24 \mathrm{~h}$. Subsequently, the mixture was treated by a probe-type Ultrasonic Processor $(20-25 \mathrm{kHz}$, Ningbo Scientz Biotechnology Co. Ltd., Ningbo, China) at $400 \mathrm{~W}$ for $10 \mathrm{~min}$ in an ice-water bath. The period of ultrasound burst was set to $2 \mathrm{~s}$ with a pause of $3 \mathrm{~s}$ between two ultrasound bursts. The resultant solution was centrifuged at $3718 \times g$ for $20 \mathrm{~min}$ to remove the free drug. Finally, the supernatant was filtered through a $0.45 \mu \mathrm{m}$ syringe filter to obtain the solution of drug-loaded nanoparticles. In addition, the nanoparticles loaded with C6 or DIR were also prepared by the same procedure for further study.

\subsection{Characterization of Silymarin-Loaded Nanoparticles}

To observe the morphology of drug-loaded nanoparticles, one drop of sample was deposited on the carbon-coated 300 mesh copper grid, air-dried and imaged using a JEM-100C transmission electron microscope (TEM, JEOL, Tokyo, Japan). Particle size and zeta potential measurements were carried out on a Nicomp 380 ZLS analyzer (PSS Nicomp, Santa Barbara, CA, USA).

Differential scanning calorimetry (DSC) was carried out using a SETSYS-1750 CS Evolution thermogravimetric analyzer (Setaram, Caluire-et-Cuire, France). Heating curves were recorded at a scan rate of $10^{\circ} \mathrm{C} / \mathrm{min}$ from 25 to $450{ }^{\circ} \mathrm{C}$ under a dry nitrogen atmosphere.

The crystalline state of SM, hm-BSP, physical mixture and drug-loaded nanoparticles were measured by an X-ray powder diffraction (XRPD) instrument (D/MARX2200/PC, Rigaku Co., Tokyo, Japan) using $\mathrm{CuK} \alpha$ radiation at $40 \mathrm{~mA}$ and $40 \mathrm{kV}$. Standard runs were performed with a scanning rate of $0.02^{\circ} / \mathrm{min}$ over a $2 \theta$ range of $3-85^{\circ}$.

The drug content was analyzed by a HPLC (Model-L2000, Hitachi, Tokyo, Japan) method [25,26]. The analytical column was a Phenomenex C18 $(5 \mu \mathrm{m}, 4.6 \times 150 \mathrm{~mm})$. The mobile phase was composed of methanol, acetonitrile and water $(16: 34: 50, v / v)$ and the final $\mathrm{pH}$ was adjusted to 4.0 with phosphoric acid, the flow rate was set at $0.8 \mathrm{~mL} / \mathrm{min}$ and the column temperature at $35^{\circ} \mathrm{C}$. The detection was performed at $226 \mathrm{~nm}$ using an UV-VIS detector (Model: Hitachi L7420).

For determination of drug content inside the nanoparticles, $200 \mu \mathrm{L}$ of sample was mixed with $10 \mathrm{~mL}$ DMSO and sonicated at $500 \mathrm{~W}$ for $30 \mathrm{~min}$. The mixtures were then filtered through a $0.22 \mu \mathrm{m}$ syringe filter, and the filtrate was used for HPLC analysis. Drug loading (DL) and encapsulation efficiency (EE) were calculated as follows:

$$
\begin{gathered}
\mathrm{DL} \%=\frac{\text { Weight of drug in micelles }}{\text { Weight of drug }- \text { loaded nanoparticles }} \times 100 \% \\
\mathrm{EE} \%=\frac{\text { Weight of drug in micelles }}{\text { Weight of feeding drug }} \times 100 \%
\end{gathered}
$$

\subsection{In Vitro Dissolution Study}

In vitro drug release studies were carried out in triplicate as follows [20]: SM-loaded nanoparticles $(10.0 \mathrm{mg})$ were introduced into a dialysis bag (molecular weight cut-off $3.4 \mathrm{kD})$ and then placed in $100 \mathrm{~mL}$ of phosphate buffer solution (PBS, pH 7.4) containing $40 \%(v / v)$ PEG 400 [27] at $37 \pm 0.5^{\circ} \mathrm{C}$ with stirring. Samples (each of $2 \mathrm{~mL}$ ) were withdrawn periodically, and then filtered through a $0.45 \mu \mathrm{m}$ syringe filter. After each withdrawal, an equal volume of the dissolution medium was added to maintain a constant volume. Drug content was determined by the HPLC method described above. 


\subsection{Cytotoxicity Test}

Human hepatoblastoma HepG2 cell lines were cultured in DMEM equilibrated with $90 \%$ humidified atmosphere of $5 \% \mathrm{CO}_{2}$ in air at $37^{\circ} \mathrm{C}$. The medium was supplemented with $10 \% \mathrm{FCS}$ and $200 \mathrm{mg} / \mathrm{L}$ SM solution. The Cell Counting Kit-8 (CCK-8) assay was used to measure cell cytotoxicity. HepG2 cells were seeded at a $3 \times 10^{4}$ cells per well in 96-well plates (Costar ${ }^{\circledR}$, Coring Inc., Coring, NY, USA) in medium and incubated for $24 \mathrm{~h}$. The medium was then replaced with $100 \mu \mathrm{L}$ of medium containing various equivalent concentrations of SM solutions or nanoparticles. The untreated cells were used as the control. The plates were incubated for another $24 \mathrm{~h}$ (or $48 \mathrm{~h}, 72 \mathrm{~h}$ ), and cytotoxicity was measured using CCK-8 kits. The absorbance was measured at a test wavelength of $450 \mathrm{~nm}$ using a microplate reader (Bio-Rad Model 550, Segrate, Italy). The percentage of cell viability (CV \%) was calculated based on the following equation:

$$
\mathrm{CV} \%=\frac{\mathrm{A}_{\text {treated }}}{\mathrm{A}_{\text {treated }}} \times 100 \%
$$

where $A_{\text {treat }}$ and $A_{\text {control }}$ were the absorbance of the treated cells and the control, respectively. Experiments were carried out in six wells and tested three times.

\subsection{Cell Uptake and Flow Cytometry Study}

The capacity for cellular internalization of C6-loaded nanoparticles was visualized and quantified by microscope and fluorometry, respectively. HepG2 cells were seeded at a density of $2 \times 10^{5}$ cells for each well in a 12-well plate $\left(\operatorname{Costar}^{\circledR}\right)$ and incubated for $24 \mathrm{~h}$. Then, cells were replenished with serum-free medium containing free $\mathrm{C} 6$ or C6-loaded nanoparticles for $1 \mathrm{~h}$. After incubation, cells were washed with ice-cooled PBS before they were fixed in 70\% ethanol. Finally, the fixed cells were observed under a fluorescence microscope (BX51TF, Olympus, Tokyo, Japan).

To quantify the cellular uptake of the fluorescence probe C6, HepG2 cells were plated at a density of a $3 \times 10^{4}$ cells per well in 12-well plates. The cells were incubated with free $\mathrm{C} 6$ or C6-hm-BSP nanoparticles in serum-free medium, washed 3 times with pH 7.4 PBS and then harvested by trypsinization. The intracellular fluorescence intensity was measured with a FACSCalibur flow cytometer (BD Biosciences, San Francisco, CA, USA). Approximately $1.0 \times 10^{4}$ cells were counted to determine the trend of micelle uptake by the HepG2 cells.

\subsection{Tissue Distribution Study}

All animal procedures were conducted in accordance with the Guidelines for the Care and Use of Laboratory Animals and were approved by the Institutional Animal Care and Use Committee at Ningxia Medical University.

To understand and compare the biodistribution of free drug and drug-loaded hm-BSP nanoparticles, a lipophilic dye (DIR) that strongly absorbs the fluorescence in the near infra-redregion [28] was encapsulated into the hm-BSP nanoparticles. The DIR nanoparticles were prepared according to the above-mentioned method at a loading capacity of $0.089 \mu \mathrm{g} / \mathrm{mL}$.

DIR-loaded nanoparticles were injected intravenously into mice via the tail vein. At $30 \mathrm{~min}$ after the injection, mice were imaged using a Kodak ${ }^{\circledR}$ in vivo imaging systemFx Pro (Carestream Health Inc., Rochester, NY, USA). The images were acquired using epiiluminationat an excitation wavelength of $730 \mathrm{~nm}$ and an emission wavelength of $790 \mathrm{~nm}$. Then, the mice were sacrificed and major organs were harvested for ex vivo imaging. The free DIR in PBS, which used Cremophor EL to solubilize at an equivalent concentration, was also injected into mice to compare the biodistribution of the DIR nanoparticles. Results were analyzed using Kodak ${ }^{\circledR}$ imaging software (Carestream Health Inc.). All experiments were repeated in three different animals and representative pictures are shown. 


\subsection{Data Statistics}

All of the results are expressed as the mean and standard deviation. The statistical analysis was performed by Student's $t$-test using SPSS statistics software (SPSS software version 16.0, IBM, Armonk, NY, USA). The $p$-value $<0.05$ was considered as statistically significant.

\section{Conclusions}

In the present study, SM was loaded into the nanoassembly of BSP conjugates with stearic acid and evaluated for the development of hepatic-targeted nanoparticles. The obtained nanoparticles appeared as spheres with an average size of $200 \mathrm{~nm}$. SM was molecularly encapsulated into the nanoparticles at a loading efficiency of $78.9 \%$ and drug loading of $7.31 \%$. The in vitro dissolution of the drug-loaded nanoparticles exhibited a typical sustained release profile. Compared to the drug solution, the developed nanoparticle formulation loaded with the drug improved cytotoxicity and cell uptake in HepG2 cell lines in vitro. These results suggest that hm-BSP derivatives are potentially effective nanocarriers for hepatic-targeted drug delivery, and the SM-hm-BSP nanoparticles will provide a better choice for enhanced clinical efficacy of SM preparations.

Acknowledgments: This work was supported by the National Natural Science Foundation of China (81160521), the Showalter Research Trust Fund, and U.S. National Institute of Health (R01GM095879).

Author Contributions: Designed and supervised experiments: Yanni Ma, Kinam Park, Wenping Wang. Performed the experiment: Yanni Ma, Shaolong He, Tongtong Hong and Zhifang Li. Analysed date: Shaolong He, Tongtong Hong, Xueqing Ma. Prepared the manuscript: Yanni Ma, Shaolong He and Wenping Wang. Reviewed and edited the manuscript: Shaolong He, Kinam Park and Wenping Wang. All authors read and approved the manuscript.

Conflicts of Interest: The authors report no conflicts of interest.

\section{References}

1. Luper, S.A. Review of Plants Used in the Treatment of Liver Disease: Part 1. Altern. Med. Rev. 1998, 3, 410-421. [PubMed]

2. Draz, E.I.; Abdin, A.A.; Sarhan, N.I.; Gabr, T.A. Neurotrophic and antioxidant effects ofsilymarin comparable to 4-methylcatechol in protection against gentamicin-induced ototoxicity in guinea pigs. Pharmacol. Rep. 2015, 67, 317-325. [CrossRef] [PubMed]

3. Amin, M.M.; Arbid, M.S. Estimation of the novel antipyretic, anti-inflammatory, antinociceptive and antihyperlipidemic effects of silymarin in Albino rats and mice. Asian Pac. J. Trop. Biomed. 2015, 5, 619-623. [CrossRef]

4. Gharagozloo, M.; Karimi, M.; Amirghofran, Z. Immunomodulatory effects of silymarin in patients with $\beta$-thalassemia major. Int. Immunopharmacol. 2013, 16, 243-247. [CrossRef] [PubMed]

5. Anthony, K.; Subramanya, G.; Uprichard, S.; Hammouda, F.; Saleh, M. Antioxidant and Anti-Hepatitis C Viral Activities of Commercial Milk Thistle Food Supplements. Antioxidants 2013, 2, 23-36. [CrossRef] [PubMed]

6. Javed, S.; Kohli, K.; Ali, M. Reassessing bioavailability of silymarin. Altern. Med. Rev. 2011, 16, $239-249$. [PubMed]

7. Rutter, K.; Scherzer, T.M.; Beinhardt, S.; Kerschner, H.; Stattermayer, A.F.; Hofer, H.; Popow-Kraupp, T.; Steindl-Munda, P.; Ferenci, P. Intravenous silibinin as "rescue treatment" for on-treatment non-respondets to pegylated interferon/ribavirin combination thetapy. Antivir. Ther. 2011, 16, 1327-1333. [CrossRef] [PubMed]

8. Christodoulou, E.; Kechagia, I.A.; Tzimas, S.; Balafas, E.; Kostomitsopoulos, N.; Archontaki, H.; Dokoumetzidis, A.; Valsami, G. Serum and tissue pharmacokinetics of silibinin after per os and i.v. administration to mice as a HP- $\beta-C D$ lyophilized product. Int. J. Pharm. 2015, 493, 366-373. [CrossRef] [PubMed]

9. Elmowafy, M.; Viitala, T.; Ibrahim, H.M.; Abu-Elyazid, S.K.; Samy, A.; Kassem, A.; Yliperttula, M. Silymarin loaded liposomes for hepatic targeting: In vitro evaluationand HepG2 drug uptake. Eur. J. Pharm. Sci. 2013, 50, 167-171. [CrossRef] [PubMed] 
10. Cho, H.; Lai, T.C.; Tomoda, K.; Kwon, G.S. Polymeric Micelles for Multi-Drug Delivery in Cancer. AAPS PharmSciTech 2015, 16, 10-20. [CrossRef] [PubMed]

11. Zhang, Y.; Li, S. Polymeric micelles: Nanocarriers for cancer-targeted drug delivery. AAPS PharmSciTech 2014, 15, 862-871. [CrossRef] [PubMed]

12. Tian, Y.; Mao, S. Amphiphilic polymeric micellesas the nanocarrier for peroraldeliveryof poorly soluble anticancer drugs. Expert Opin. Drug Deliv. 2012, 9, 687-700. [CrossRef] [PubMed]

13. Chen, M.; Liu, Y.; Yang, W.; Li, X.; Liu, L.; Zhou, Z.; Wang, Y.; Li, R.; Zhang, Q. Preparation and characterization of self-assembled nanoparticles of 6-O-cholesterol-modified chitosan for drug delivery. Carbohydr. Polym. 2011, 8, 1244-1251. [CrossRef]

14. Platt, V.M.; Szoka, F.C., Jr. Anticancer Therapeutics: Targeting Macromolecules and anocarriers to Hyaluronan or CD44, a Hyaluronan Receptor. Mol. Pharm. 2008, 5, 474-486. [CrossRef] [PubMed]

15. Liu, Z.; Jiao, Y.; Wang, Y.; Zhou, C.; Zhang, Z. Polysaccharides-based nanoparticles as drug delivery systems. Adv. Drug Deliv. Rev. 2008, 60, 1650-1662. [CrossRef] [PubMed]

16. Wang, W.; He, S.; Hong, T.; Zhang, Y.; Sui, H.; Zhang, X.; Ma, Y. Synthesis, self-assembly, and in vitro toxicity of fatty acids-modified Bletilla striata polysaccharide. Aritif. Cells Nanamed. Biotechnol. 2016, 11. [CrossRef]

17. Jia, L.; Zhang, D.; Li, Z.; Duan, C.; Wang, Y.; Feng, F.; Wang, F.; Liu, Y.; Zhang, Q. Nanostructured lipid carriers for parenteral delivery of silybin: Biodistributionand pharmacokinetic studies. Colloids Surf. B Biointerfaces 2010, 80, 213-218. [CrossRef] [PubMed]

18. Lian, R.; Lu, Y.; Qi, J.; Tan, Y.; Niu, M.; Guan, P.; Hu, F.; Wu, W. Silymarin Glyceryl Monooleate/Poloxamer 407 Liquid Crystalline Matrices: Physical Characterization and Enhanced Oral Bioavailability. AAPS PharmSciTech 2011, 12, 1234-1240. [CrossRef] [PubMed]

19. Yang, W.; Wang, M.; Ma, L.; Li, H.; Huang, L. Synthesis and characterization of biotin modified cholesteryl pullulanas a novel anticancer drug carrier. Carbohydr. Polym. 2014, 99, 720-727. [CrossRef] [PubMed]

20. Wang, Y.; Yang, X.; Yang, J.; Wang, Y.; Chen, R.; Wu, J.; Liu, Y.; Zhang, N. Self-assembled nanoparticles of methotrexate conjugated $O$-carboxymethylchitosan: Preparation, characterization and drug release behavior in vitro. Carbohydr. Polym. 2011, 86, 1665-1670. [CrossRef]

21. Yang, J.; Liu, Y.; Wang, H.; Liu, L.; Wang, W.; Wang, C.; Wang, Q.; Liu, W. The biocompatibility of fatty acid modified dextran-agmatine bioconjugate gene delivery vector. Biomaterials 2012, 33, 604-613. [CrossRef] [PubMed]

22. Sallustio, S.; Galantini, L.; Gente, G.; Masci, G.; la Mesa, C. Hydrophobically Modified Pullulans: Characterization and Physicochemical Properties. J. Phys. Chem. 2004, 108, 18876-18883. [CrossRef]

23. Liu, Y.; Sun, J.; Zhang, P.; He, Z. Amphiphilic Polysaccharide-Hydrophobicized Graft Polymeric Micelles for Drug Delivery Nanosystems. Curr. Med. Chem. 2011, 18, 2638-2648. [CrossRef] [PubMed]

24. Ye, Y.Q.; Yang, F.L.; Hu, F.Q.; Du, Y.Z.; Yuan, H.; Yu, H.Y. Core-modified chitosan-based polymeric micelles for controlled release of doxorubicin. Int. J. Pharm. 2008, 352, 294-301. [CrossRef] [PubMed]

25. Radjadian, T.; Rezazadeh, S.H.; Huseini, H.F. Analysis of silymarin components in the seed extracts of some milk thistle ecotypes from Iran by HPLC. Iran. J. Sci. Technol. A 2008, 32, 141-146.

26. Usman, M.; Ahmad, M.; Dayo, A.; Madni, A.; Ali, L.; Yousuf, M.; Khan, M.A.; Munir, A.; Sohail, M.; Mahmood, A. Effect of $\beta$-Glucuronidase on Extraction Efficiency of Silymarin from Human Plasma Samples Using Validated HPLC-UV Analysis. Trop. J. Pharm. Res. 2012, 11, 84-90. [CrossRef]

27. Shakeel, F; Anwer, M.K. Dissolution thermodynamics and solubility of silymarin in PEG 400-water mixtures at different temperatures. Drug Dev. Ind. Pharm. 2015, 41, 1819-1823. [CrossRef] [PubMed]

28. Zhang, X.; Guo, S.; Fan, R.; Yu, M.; Li, F.; Zhu, C.; Gan, Y. Dual-functional liposome for tumor targeting and overcoming multidrug resistance in hepatocellular carcinoma cells. Biomaterials 2012, 33, 7103-7114. [CrossRef] [PubMed]

Sample Availability: Samples of the nanoparticles are available from the authors.

(C) 2016 by the authors; licensee MDPI, Basel, Switzerland. This article is an open access article distributed under the terms and conditions of the Creative Commons by Attribution (CC-BY) license (http:/ / creativecommons.org/licenses/by/4.0/). 